\title{
Positive and negative regulators of the metallothionein gene (Review)
}

\author{
SHINICHIRO TAKAHASHI
}

\begin{abstract}
Division of Molecular Hematology, Kitasato University Graduate School of Medical Sciences and Division of Hematology, Kitasato University School of Allied Health Sciences, Sagamihara, Kanagawa 252-0373, Japan
\end{abstract}

Received July 11, 2014; Accepted February 26, 2015

DOI: $10.3892 / \mathrm{mmr} .2015 .3459$

\begin{abstract}
Metallothioneins (MTs) are metal-binding proteins involved in diverse processes, including metal homeostasis and detoxification, the oxidative stress response and cell proliferation. Aberrant expression and silencing of these genes are important in a number of diseases. Several positive regulators of $M T$ genes, including metal-responsive element-binding transcription factor (MTF)-1 and upstream stimulatory factor (USF)-1, have been identified and mechanisms of induction have been well described. However, the negative regulators of $M T$ genes remain to be elucidated. Previous studies from the group of the present review have revealed that the hematopoietic master transcription factor, PU.1, directly represses the expression levels of $M T$ genes through its epigenetic activities, and upregulation of $M T$ results in the potent inhibition of myeloid differentiation. The present review focuses on PU.1 and several other negative regulators of this gene, including PZ120, DNA methyltransferase 3a with Mbd3 and Brg1 complex, CCAAT enhancer binding protein $\alpha$ and Ku protein, and describes the suppression of the MT genes through these transcription factors.
\end{abstract}

\section{Contents}

1. Introduction

2. Positive regulators of $M T$ genes

3. Negative regulators of $M T$ genes

4. PU.1-a master hematopoietic transcription factor previously identified as a novel negative regulator of $M T-1 \mathrm{~s}$

5. Conclusion

Correspondence to: Professor Shinichiro Takahashi, Division of Molecular Hematology, Kitasato University Graduate School of Medical Sciences and Division of Hematology, Kitasato University School of Allied Health Sciences, 1-15-1 Kitasato, Sagamihara, Kanagawa 252-0373, Japan

E-mail: shin@kitasato-u.ac.jp

Key words: metallothionein, regulation, PU.1

\section{Introduction}

The metallothioneins (MTs) are a group of low molecular weight, cysteine-rich intracellular proteins, which are involved in maintaining intracellular metal homeostasis by binding metals, including zinc and copper. There are 10 functional isoforms of MTs, which are divided into four classes, designated MT-1 to -4 , on the basis of small differences in protein sequence, expression and characteristics $(1,2)$. They maintain transition metal ion homeostasis and redox balance, serve as anti-oxidants and protect against DNA damage and apoptosis (3). Reduced expression of $M T$ has been observed in liver (4), colon (5) and prostate (6) cancer. It was suggested that during the transformation of normal colorectal tissue to adenomatous polyps and adenocarcinoma, a progressive decrease in the expression of $M T$ occurs $(7,8)$. The role of $M T$ in these types of cancer remains to be elucidated, however, considering its anti-oxidant activity and its protective potential against DNA damage, this reduction may increase susceptibility to toxin-induced damage. Indeed, an $M T$ knockout in mice has been reported to induce a higher rate of induced carcinogenesis (9). Conversely, aberrant overexpression of $M T$ has been observed in various types of human cancer, including breast cancer, gallbladder cancer, melanoma and lymphoma (10-13). It has been suggested that the overexpression of MT may protect cells from free radical-induced DNA damage and lipid peroxidation (14). Overexpression of $M T$ has been demonstrated to be important in drug resistance, since nuclear expression of $M T$ protects DNA in ovarian cancer cells from the toxic effect of treatment with cisplatin (15). This indicates that aberrant under/over-expression of $M T$ are important in various types of cancer.

A study revealed that the hematopoietic master transcription factor, PU.1, directly suppresses the $M T-1 A$ and $M T-1 G$ promoter through DNA methylation and histone deacetylase (HDAC) activity (16). Additionally, it was revealed that $M T-1 A$ is suppressed, while the expression of PU.1 is induced, during 12-O-tetradecanoylphorbol-13-acetate (TPA)-induced monocytic differentiation of THP-1 cells (17). Notably, the suppression of $M T-1 s$ by PU.1 is required for the proper differentiation of myeloid cells.

Although there are several reviews regarding the regulation of the $M T$ gene (18-20), reviews regarding the suppressive regulation of $M T$ genes are relatively scarce. Therefore, this 
review summarized the regulation of $M T$ genes and particularly focused on PU.1 and other suppressive regulators of the $M T$ genes.

\section{Positive regulators of $M T$ genes}

The basal activity of $M T$ is regulated by several general transcription factors, including the TFIID complex comprising TATA-binding protein (TBP), TBP associated factors and Sp1 (18-20). In addition, $M T$ can be activated by a variety of stimuli, including metal ions, cytokines and growth factors (1). Several inducible expression regulators of the $M T$ genes have been identified, including metal-responsive element (MRE)-binding transcription factor (MTF)-1 $(21,22)$, upstream stimulatory factor (USF)-1 (23) and nuclear factor (NF)1 (24). Since a number of reviews summarize the details of the positive regulation of $M T$ genes (18-20), the present review describes the above essential factors.

The $M T F-1$ gene is a central regulator of the metal-inducible expression levels of $M T-1$ and $M T-2$. In addition to zinc, other heavy metals (e.g. cadmium), hypoxia, oxidative stress, stress hormones (glucocorticoids), nitric oxide and high temperature induce the transcriptional activity of MTF-1 (25-28). Andrews et al (23) reported that MTF-1 is essential for the upregulation of the gene expression of MT-1 in visceral endoderm cells and that optimal expression is dependent upon the interactions of the basic helix-loop-helix transcription factor, USF -1, with an E-box-1 containing sequence at -223 bp in the $M T-1$ promoter (23).

NF1 is a protein expressed ubiquitously in higher eukaryotes, and distinct highly conserved genes encode four isoforms of the NF1 protein (NF1-A, NF1-B, NF1-C and NF1-X) (29-31). NF1 binding sites were identified in various $M T$ promoters, with the exception of MT-IB (19). LaRochelle et al (24) previously demonstrated that NF1 binds to the mouse $M T-1$ promoter in vivo and this binding is zinc inducible and MTF-1 dependent. It was revealed by transient transfection assays into HepG2 cells, that NF1 activates the mouse $M T-1$ promoter. The authors demonstrated that NF1 and MTF-1 synergistically activate the mouse MT-1 gene in response to metal ions (24). However, Majumder et al $(32,33)$ previously demonstrated that NF1 isoforms inhibit the activity of the $M T-1$ promoter in HepG2 cells. This is contradictory to the earlier study (24), however, this result may be due to the experimental condition in which Majumder et al have used extremely high expression levels of the NF1 vector, 30- to 1000-fold more vector compared with the earlier study (24). LaRochelle et al demonstrated that the expression levels of the transcriptionally active mutant of NF1 reduced the zinc-induced $M T-1$ promoter by up to $50 \%$, in a dose-dependent manner and may also indicate that NF1 is a positive regulator of the gene expression of MT-1 (24).

\section{Negative regulators of $M T$ genes}

To date, several factors are reported to regulate the suppression of MT genes, including PZ120 (34), DNA methyltransferase (Dnmt) 3a with Mbd3 and Brg1 complex (35), C/EBP $\alpha$ (36), Ku protein (37) and PU.1 (16,17).
Tang et al (34), reported the cloning of a novel zinc finger protein with a molecular mass of $120 \mathrm{kDa}$ (PZ120), through Southwestern cloning, which interacts specifically with the human gene transcription initiation site of $M T-2 A$. PZ120 is a ubiquitously expressed protein and possesses a conserved poxvirus and zinc finger (POZ) motif, which is a structure existing in several transcriptional repressors. This protein has been revealed to repress the transcription of the $M T-2 A$ promoter (34).

Datta et al (35) purified DNA methyltransferase (Dnmt) 3a from mouse lymphosarcoma cells and revealed that Dnmt 3a-associated polypeptides identified the methyl $\mathrm{CpG}$ binding protein, Mbd3, histone deacetylase 1 and components of the Brg1 complex (35). A chromatin immunoprecipitation assay reveled that Dnmt 3a, Mbd3 and Brg1 are associated with a transcriptionally silent methylated $M T-1$ promoter in the mouse lymphosarcoma cells. The authors further clarified that the catalytic activity of Dnmt3a was not important for the repression of the MT-1 gene; however, ATP-dependent chromatin remodeling of Brg1 was (35). It was also revealed that methylated and unmethylated $M T-1$ promoters are differentially regulated by several methyl $\mathrm{CpG}$ binding proteins, including methyl $\mathrm{CpG}$ binding protein (MeCP) 2 and Mbd1, 2 and 4 (38).

CCAAT enhancer binding protein $(\mathrm{C} / \mathrm{EBP})$ is important in the terminal differentiation of cells, particularly in myeloid cells and adipose cells (39). Yin et al (36) demonstrated that forced expression of $\mathrm{C} / \mathrm{EBP} \alpha$ decreased the expression levels of the $M T$ isoforms $1 \mathrm{~A}, \mathrm{~B}, \mathrm{~F}$ and $\mathrm{H}$, and $2 \mathrm{~A}$ and 3 in prostate cancer cells, and that this suppression is mediated through its promoter activity. Furthermore, it was revealed that the forced expression of $\mathrm{C} / \mathrm{EBP} \alpha$ led to an increased cytotoxicity of zinc in prostate cancer cells (36). However, in human hepatocellular carcinoma cells, the inactivation of $\mathrm{C} / \mathrm{EBP} \alpha$ through the activation of phosphatidylinositol 3-kinase led to the downregulation of the expression of $M T$ (4). Therefore, the role of $\mathrm{C} / \mathrm{EBP} \alpha$ in the gene regulation of $M T$ may differ among tissues.

It was previously reported that the large subunit (p80) of the $\mathrm{Ku}$ protein contained repressor activity for the MT-1 promoter (37). Additionally, it was revealed that this repression is due to the hypermethylation of a $\mathrm{CpG}$ island in the $M T-1$ promoter (40).

Rodent and human $M T$ genes contain $\mathrm{CpG}$ islands in their promoter $(19,41)$. It was first reported in 1981 that DNA methylation controls the inducibility of the mouse $M T-1$ gene (42). Since then, $>100$ studies have been published demonstrating that the $M T$ promoter is regulated by DNA methylation in its promoter region. Arriaga et al (43) demonstrated from the analysis of colorectal cancer, that the mRNA expression levels of five isoforms $(M T-1 G, 1 E, 1 F, 1 H$ and $1 M$ ) were lost during the transition from normal mucosa to tumor, whereas $M T-1 X$ and $M T-2$ were less downregulated and their expression was correlated with overall protein positivity. It was also demonstrated that hypermethylation of the $M T-1 G$ gene occurred in cell lines and in $29 \%$ of tumor samples. Faller et al (44) analyzed specimens from patients with melanoma and demonstrated that in $1 / 17(6 \%)$ of the benign naevi, $16 / 43(37 \%)$ primary tumors and 6/13 (46\%) of metastases exhibited $M T-1 E$ gene methylation. Peng et al (45) revealed 


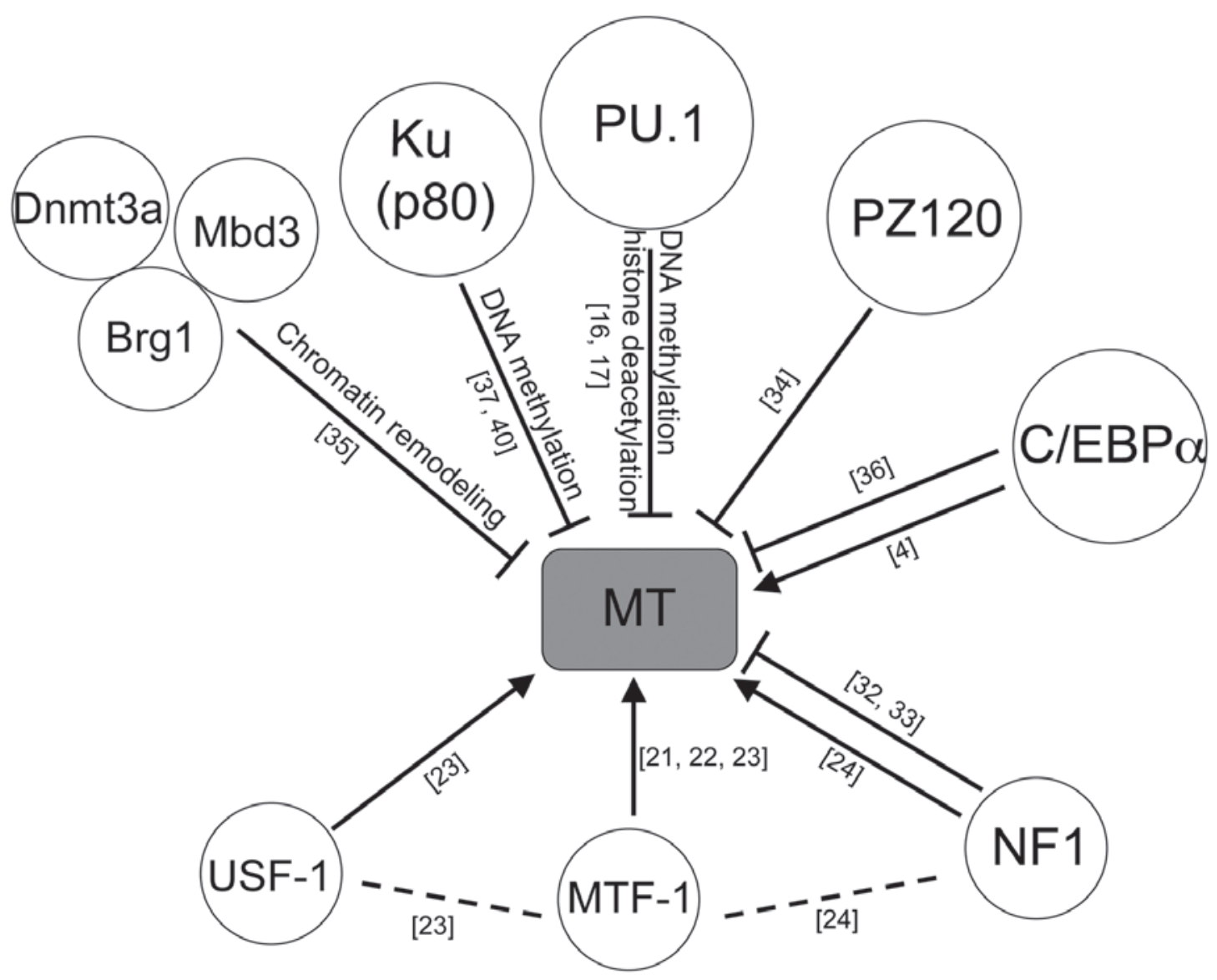

Figure 1. Schematic presentation of the negative and positive regulators of $M T$. Dotted line indicates a possible interaction. The indicated numbers in brackets are the references cited in this review. MT, metallothionein; Dnmt3a, DNA methyltransferase 3a; Mbd3, methyl-CpG-binding domain protein 3; PZ120, zinc finger protein with a molecular weight of $120 \mathrm{kDa} ; \mathrm{C} / \mathrm{EBP} \alpha, \mathrm{CCAAT}$-enhancer-binding protein $\alpha ; \mathrm{NF} 1$, nuclear factor $1 ;$ MTF-1, metal regulatory transcription factor 1; USF-1, upstream transcription factor 1.

using quantitative pyrosequencing, unique DNA methylation profiles in the $M T-3$ promoter region in esophageal adenocarcinomas (EACs). This previous study concluded that EACs are characterized by frequent epigenetic silencing of the $M T-3$ gene. In addition, in colon cancer, not only DNA methylation $(41,43)$, but the loss of heterozygosity (5) is also important in the downregulation of the $M T$ genes $(M T-1 \mathrm{~F}$, $M T-1 \mathrm{G}, M T-1 \mathrm{X}$ and $M T-2 \mathrm{~A})$.

\section{PU.1-a master hematopoietic transcription factor previously identified as a novel negative regulator of MT-1s}

A previous study revealed that $M T-1 s$ genes are epigenetically suppressed by the activity of PU.1 (16). PU.1 is a hematopoietic master transcription factor, predominantly expressed in immature myeloid cells and B cells, and downregulation of this factor is important in various hematological malignancies $(46,47)$. To identify downstream target genes of PU.1, the authors generated cell lines expressing reduced levels of PU.1 by stable transfection of PU.1 short inhibitory RNAs into K562 human myeloid leukemia cells (K562PU.1KD cells) and PU.1-overexpressing K562 cells (K562PU.1OE cells). Dual microarray analyses were performed using these cell lines.
Notably, the expression levels of all the functional $M T$ isoforms expressed in humans $(M T-1 A,-B,-E,-F,-G,-H$ and $-X$ and $M T-2)$ were increased by varying degrees in the K562PU.1KD cells. Furthermore, there were negative correlations between the mRNA expression of PU.1 and the mRNA expression of the $M T-1 s$ in 43 primary specimens from patients with acute myeloid leukemia (AML). Additionally, it was revealed that PU.1 directly binds and epigenetically suppresses the MT-1s promoter, in concert with $\mathrm{MeCP} 2$, through the suppression of the enzymatic activities of HDAC and Dnmt. The proportion of the methylated $\mathrm{CpG}$ sites is tightly associated with the expression levels in $M T-1 s$ promoters (16). Next, the authors examined whether the expression levels of PU.1 and MT-1A are indeed correlated with each other, and whether the expression of $M T-1 A$ is regulated by PU.1 during TPA-induced THP-1 monocyte differentiation. As a result, it was revealed that the expression of $M T-1 s$ is suppressed during monocytic differentiation in the THP-1 cells (17). Chromatin immunoprecipitation analysis demonstrated that PU.1 and MeCP2 bind to the same region in the $M T-1 A$ promoter, and the binding of these proteins to this promoter was increased during differentiation. Consistently, the proportion of methylated $\mathrm{CpG}$ sites was markedly increased during differentiation (17). These results suggest that $M T-1 s$ are repressed through the epigenetic activity of PU.1 in hematopoietic cells. 


\section{Conclusion}

The positive and negative regulators described in this review are summarized in Fig. 1. The consequences of these $M T$ gene regulations have been reported to be through the normal physiological aspects to disease, including inflammation, aging and malignancies $(1,3,48,49)$. It was recently demonstrated that the overexpression of $M T-1 G$ potently inhibited the retinoic acid induced myeloid differentiation of NB4 acute promyelocytic leukemia cells (50). This is consistent with the literature, suggesting that the downregulation of PU.1 is the cause of AML (46) and results in the overexpression of $M T$, leading to the inhibition of differentiation, which is important in leukemogenesis.

$M T$ s are multifunctional proteins and exhibit different biological behavior in different tissues. Therefore, further clarifying the underlying mechanisms and the roles of $M T$, may lead to an improved understanding of the biology of normal physiology and malignancies from another aspects.

\section{Acknowledgements}

The author would like to thank everyone who helped the research during this decade, regarding the analysis of the PU.1 transcription factor, and the regulation and functions of the $M T$ gene. This study was supported in part by Grants-in-Aid for Scientific Research (grant no. 26460685) from the Ministry of Education, Culture, Sports, Science and Technology, Japan, the Takeda Science Foundation, and a foundation from Kitasato University School of Allied Health Sciences (Grant-in-Aid for Research Project, grant no. 2014-1003).

\section{References}

1. Takahashi S: Molecular functions of metallothionein and its role in hematological malignancies. J Hematol Oncol 5: 41, 2012.

2. Hamer DH: Metallothionein. Annu Rev Biochem 55: 913-951, 1986.

3. Cherian MG, Jayasurya A and Bay BH: Metallothioneins in human tumors and potential roles in carcinogenesis. Mutat Res 533: 201-209, 2003.

4. Datta J, Majumder S, Kutay H, et al: Metallothionein expression is suppressed in primary human hepatocellular carcinomas and is mediated through inactivation of CCAAT/enhancer binding protein alpha by phosphatidylinositol 3-kinase signaling cascade. Cancer Res 67: 2736-2746, 2007.

5. Yan DW, Fan JW, Yu ZH, et al: Downregulation of metallothionein $1 \mathrm{~F}$, a putative oncosuppressor, by loss of heterozygosity in colon cancer tissue. Biochim Biophys Acta 1822: 918-926, 2012.

6. Wei H, Desouki MM, Lin S, Xiao D, Franklin RB and Feng P: Differential expression of metallothioneins (MTs) 1, 2, and 3 in response to zinc treatment in human prostate normal and malignant cells and tissues. Mol Cancer 7: 7, 2008.

7. Theocharis SE, Margeli AP, Klijanienko JT and Kouraklis GP: Metallothionein expression in human neoplasia. Histopathology 45: 103-118, 2004.

8. Ofner D, Maier H, Riedmann B, et al: Immunohistochemical metallothionein expression in colorectal adenocarcinoma: correlation with tumour stage and patient survival. Virchows Arch 425: 491-497, 1994

9. Zhang B, Satoh M, Nishimura N, et al: Metallothionein deficiency promotes mouse skin carcinogenesis induced by 7,12-dimethylbenz[a]anthracene. Cancer Res 58: 4044-4046, 1998.

10. Poulsen CB, Borup R, Borregaard N, Nielsen FC, Møller MB and Ralfkiaer E: Prognostic significance of metallothionein in B-cell lymphomas. Blood 108: 3514-3519, 2006.
11. Bay BH, Jin R, Huang J and Tan PH: Metallothionein as a prognostic biomarker in breast cancer. Exp Biol Med (Maywood) 231: 1516-1521, 2006

12. Shukla VK, Aryya NC, Pitale A, et al: Metallothionein expression in carcinoma of the gallbladder. Histopathology 33: 154-157, 1998

13. Weinlich G, Eisendle K, Hassler E, Baltaci M, Fritsch PO and Zelger B: Metallothionein-overexpression as a highly significant prognostic factor in melanoma: a prospective study on 1270 patients. Br J Cancer 94: 835-841, 2006.

14. You HJ, Lee KJ and Jeong HG: Overexpression of human metallothionein-III prevents hydrogen peroxide-induced oxidative stress in human fibroblasts. FEBS Lett 521: 175-179, 2002.

15. Surowiak P, Materna V, Maciejczyk A, et al: Nuclear metallothionein expression correlates with cisplatin resistance of ovarian cancer cells and poor clinical outcome. Virchows Arch 450: 279-285, 2007.

16. Imoto A, Okada M, Okazaki T, Kitasato H, Harigae $\mathrm{H}$ and Takahashi S: Metallothionein-1 isoforms and vimentin are direct PU.1 downstream target genes in leukemia cells. J Biol Chem 285: 10300-10309, 2010.

17. Suzuki S, Nakano H and Takahashi S: Epigenetic regulation of the metallothionein-1A promoter by PU.1 during differentiation of THP-1 cells. Biochem Biophys Res Commun 433: 349-353, 2013.

18. Ghoshal K and Jacob ST: Regulation of metallothionein gene expression. Prog Nucleic Acid Res Mol Biol 66: 357-384, 2001.

19. Laukens D, Waeytens A, De Bleser P, Cuvelier C and De Vos M: Human metallothionein expression under normal and pathological conditions: mechanisms of gene regulation based on in silico promoter analysis. Crit Rev Eukaryot Gene Expr 19: 301-317, 2009.

20. Haq F, Mahoney M and Koropatnick J: Signaling events for metallothionein induction. Mutat Res 533: 211-226, 2003.

21. Radtke F, Heuchel R, Georgiev O, et al: Cloned transcription factor $M T F-1$ activates the mouse metallothionein I promoter. EMBO J 12: 1355-1362, 1993.

22. Palmiter RD: Regulation of metallothionein genes by heavy metals appears to be mediated by a zinc-sensitive inhibitor that interacts with a constitutively active transcription factor, $M T F-1$. Proc Natl Acad Sci USA 91: 1219-1223, 1994.

23. Andrews GK, Lee DK, Ravindra R, et al: The transcription factors $M T F-1$ and USF1 cooperate to regulate mouse metallothionein-I expression in response to the essential metal zinc in visceral endoderm cells during early development. EMBO J 20: 1114-1122, 2001.

24. LaRochelle O, Labbe S, Harrisson JF, et al: Nuclear factor-1 and metal transcription factor-1 synergistically activate the mouse metallothionein-1 gene in response to metal ions. J Biol Chem 283: 8190-8201, 2008

25. Westin G and Schaffner W: A zinc-responsive factor interacts with a metal-regulated enhancer element (MRE) of the mouse metallothionein-I gene. EMBO J 7: 3763-3770, 1988.

26. Dalton T, Palmiter RD and Andrews GK: Transcriptional induction of the mouse metallothionein-I gene in hydrogen peroxide-treated Hepa cells involves a composite major late transcription factor/antioxidant response element and metal response promoter elements. Nucleic Acids Res 22: 5016-5023, 1994.

27. Murphy BJ, Andrews GK, Bittel D, et al: Activation of metallothionein gene expression by hypoxia involves metal response elements and metal transcription factor-1. Cancer Res 59: 1315-1322, 1999.

28. Günther V, Lindert U and Schaffner W: The taste of heavy metals: gene regulation by $M T F-1$. Biochim Biophys Acta 1823: $1416-1425,2012$.

29. Paonessa G, Gounari F, Frank R and Cortese R: Purification of a NF1-like DNA-binding protein from rat liver and cloning of the corresponding cDNA. EMBO J 7: 3115-3123, 1988.

30. Rupp RA, Kruse U, Multhaup G, Göbel U, Beyreuther K and Sippel AE: Chicken NFI/TGGCA proteins are encoded by at least three independent genes: NFI-A, NFI-B and NFI-C with homologues in mammalian genomes. Nucleic Acids Res 18: 2607-2616, 1990

31. Meisterernst M, Rogge L, Foeckler R, Karaghiosoff M and Winnacker EL: Structural and functional organization of a porcine gene coding for nuclear factor I. Biochemistry 28: 8191-8200, 1989.

32. Majumder S, Ghoshal K, Gronostajski RM and Jacob ST: Downregulation of constitutive and heavy metal-induced metallothionein-I expression by nuclear factor I. Gene Expr 9: 203-215, 2001. 
33. Jacob ST, Majumder S and Ghoshal K: Suppression of metallothionein-I/II expression and its probable molecular mechanisms. Environ Health Perspect 110 (Suppl 5): 827-830, 2002.

34. Tang CM, Westling J and Seto E: trans repression of the human metallothionein IIA gene promoter by PZ120, a novel 120-kilodalton zinc finger protein. Mol Cell Biol 19: 680-689, 1999.

35. Datta J, Majumder S, Bai S, et al: Physical and functional interaction of DNA methyltransferase 3A with Mbd3 and Brg1 in mouse lymphosarcoma cells. Cancer Res 65: 10891-10900, 2005.

36. Yin H, Smith $M$ and Glass J: Stable expression of C/EBPalpha in prostate cancer cells down-regulates metallothionein and increases zinc-induced toxicity. Prostate 62: 209-216, 2005.

37. Ghoshal K, Li Z and Jacob ST: Overexpression of the large subunit of the protein $\mathrm{Ku}$ suppresses metallothionein-I induction by heavy metals. Proc Natl Acad Sci USA 95: 10390-10395, 1998

38. Majumder S, Kutay H, Datta J, Summers D, Jacob ST and Ghoshal K: Epigenetic regulation of metallothionein-i gene expression: differential regulation of methylated and unmethylated promoters by DNA methyltransferases and methyl CpG binding proteins. J Cell Biochem 97: 1300-1316, 2006.

39. Koschmieder S, Halmos B, Levantini E and Tenen DG: Dysregulation of the C/EBPalpha differentiation pathway in human cancer. J Clin Oncol 27: 619-628, 2009.

40. Majumder S, Ghoshal K, Li Z and Jacob ST: Hypermethylation of metallothionein-I promoter and suppression of its induction in cell lines overexpressing the large subunit of $\mathrm{Ku}$ protein. J Biol Chem 274: 28584-28589, 1999.

41. Mao J, Yu H, Wang C, et al: Metallothionein $M T 1 \mathrm{M}$ is a tumor suppressor of human hepatocellular carcinomas. Carcinogenesis 33: 2568-2577, 2012.
42. Compere SJ and Palmiter RD: DNA methylation controls the inducibility of the mouse metallothionein-I gene lymphoid cells. Cell 25: 233-240, 1981.

43. Arriaga JM, Levy EM, Bravo AI, et al: Metallothionein expression in colorectal cancer: relevance of different isoforms for tumor progression and patient survival. Hum Pathol 43: 197-208, 2012.

44. Faller WJ, Rafferty M, Hegarty S, et al: Metallothionein 1E is methylated in malignant melanoma and increases sensitivity to cisplatin-induced apoptosis. Melanoma Res 20: 392-400, 2010.

45. Peng D, Hu TL, Jiang A, et al: Location-specific epigenetic regulation of the metallothionein 3 gene in esophageal adenocarcinomas. PLoS One 6: e22009, 2011.

46. Rosenbauer F, Wagner K, Kutok JL, et al: Acute myeloid leukemia induced by graded reduction of a lineage-specific transcription factor, PU.1. Nat Genet 36: 624-630. Epub 2004 May 2016., 2004.

47. Pettersson M, Sundström C, Nilsson K and Larsson LG: The hematopoietic transcription factor PU.1 is downregulated in human multiple myeloma cell lines. Blood 86: 2747-2753, 1995.

48. Inoue $\mathrm{K}$ and Takano $\mathrm{H}$ : Metallothionein as a negative regulator of pulmonary inflammation. Curr Pharm Biotechnol 14: 414-419, 2013.

49. Mocchegiani E, Costarelli L, Basso A, Giacconi R, Piacenza F and Malavolta M: Metallothioneins, ageing and cellular senescence: a future therapeutic target. Curr Pharm Des 19: 1753-1764, 2013.

50. Hirako N, Nakano H and Takahashi S: A PU.1 suppressive target gene, metallothionein $1 \mathrm{G}$, inhibits retinoic acid-induced NB4 cell differentiation PLoS One 9: e103282, 2014. 